\title{
In the absence of renal disease, 20 year mortality risk in type 1 diabetes is comparable to that of the general population: a report from the Pittsburgh Epidemiology of Diabetes Complications Study
}

\author{
T. J. Orchard • A. M. Secrest • R. G. Miller • T. Costacou
}

Received: 7 April 2010 /Accepted: 6 July 2010 /Published online: 28 July 2010

(C) Springer-Verlag 2010

\begin{abstract}
Aims/hypothesis The FinnDiane Study has reported that mortality in type 1 diabetes is not increased over a 7 year follow-up in the absence of renal disease (RD). Using the Pittsburgh Epidemiology of Diabetes Complications (EDC) Study population $(n=658)$ of childhood-onset type 1 diabetes (age $<17$ years), the present study sought to replicate and expand these findings to a 20 year follow-up (as of 1 January 2008) and examine cause of death by renal status.

Methods At baseline (1986-1988), mean age and duration of diabetes were 28 and 19 years, respectively. RD was defined as an albumin excretion rate $\geq 20 \mu \mathrm{g} / \mathrm{min}$ from multiple samples and grouped as microalbuminuria (MA; 20-200 $\mu \mathrm{g} / \mathrm{min}$ ), overt nephropathy (ON; $>200 \mu \mathrm{g} / \mathrm{min})$, or end stage renal disease (ESRD; dialysis or renal transplantation).

Results At baseline, 311 (47.3\%) individuals had RD (MA $21.3 \%$, ON $22.2 \%$ and ESRD $3.8 \%$ ). During a median 20 year follow-up, there were 152 deaths (23.1\%). Mortality was $6.2(95 \%$ CI $5.2-7.2)$ times higher than expected, with standardised mortality ratios of $2.0(1.2-2.8)$ for normoalbuminuria (NA); 6.4 (4.4-8.4) for MA; 12.5 (9.5-15.4) for ON; and $29.8(16.8-42.9)$ for ESRD. Excluding those $(n=64)$ with NA who later progressed to $\mathrm{RD}$, no significant excess mortality was observed in the remaining NA group $(1.2,0.5-1.9)$, whose deaths were largely unrelated to diabetes.
\end{abstract}

T. J. Orchard $(\bowtie) \cdot$ A. M. Secrest $\cdot$ R. G. Miller $\cdot$ T. Costacou Department of Epidemiology, Graduate School of Public Health, University of Pittsburgh,

3512 Fifth Avenue, 2nd Floor,

Pittsburgh, PA 15213, USA

e-mail: tjo@pitt.edu
Conclusions/interpretation These data confirm the importance of $\mathrm{RD}$, including persistent microalbuminuria, as a marker of mortality risk and suggest that type 1 diabetes patients without renal disease achieve long-term survival comparable to the general population.

Keywords End stage renal disease $\cdot$ Microalbuminuria . Mortality - Overt nephropathy $\cdot$ Renal disease $\cdot$ Type 1 diabetes

$\begin{array}{ll}\text { Abbreviations } \\ \text { EDC } & \text { Epidemiology of Diabetes Complications } \\ \text { eGDR } & \text { Estimated glucose disposal rate } \\ \text { eGFR } & \text { Estimated glomerular filtration rate } \\ \text { ESRD } & \text { End stage renal disease } \\ \text { HDL-c } & \text { HDL-cholesterol } \\ \text { MA } & \text { Microalbuminuria } \\ \text { MDRD } & \text { Modification of diet in renal disease } \\ \text { NA } & \text { Normoalbuminuria } \\ \text { ON } & \text { Overt nephropathy } \\ \text { RD } & \text { Renal disease } \\ \text { SBP } & \text { Systolic blood pressure } \\ \text { SMR } & \text { Standardised mortality ratio } \\ \text { WBC } & \text { White blood cell }\end{array}$

\section{Introduction}

Many previous studies have documented that individuals with type 1 diabetes have an increased age-adjusted mortality risk of death compared with the general population $[1,2]$. This excess mortality risk results in large part from the development of chronic complications, particularly renal disease (RD) [3-7]. Furthermore, a recent article by 
Groop et al. (FinnDiane Study) reports that, while 7 year mortality in type 1 diabetes patients increases in a stepwise manner by degree of albuminuria at baseline, individuals with normoalbuminuria have an equivalent mortality to that of the general age and sex-matched Finnish population [8].

Despite these landmark FinnDiane findings, four salient questions remain unanswered. First, it is unknown whether the FinnDiane findings are unique to the Finnish population and its medical system. Second, as the analysis by Groop et al. covered a median follow-up of only 7 years, whether these findings will also apply over a longer time span is unknown. Similarly, the contemporary long-term mortality of individuals with persistent normoalbuminuria is not well documented. Finally, it is not known whether, as would be predicted by these findings, individuals with type 1 diabetes and normoalbuminuria die only from non-diabetes-related causes. Using 20 years of follow-up data from a well-characterised hospitalbased cohort of individuals with type 1 diabetes living near Pittsburgh, Pennsylvania, USA, we sought to replicate the FinnDiane findings and answer these questions.

\section{Methods}

Study population The Pittsburgh Epidemiology of Diabetes Complications (EDC) Study has previously been described in detail $[9,10]$. The EDC population has been shown to be epidemiologically representative of the type 1 diabetes population in Allegheny County, Pennsylvania [11]. Briefly, Pittsburgh EDC Study participants $(n=658)$, who were diagnosed with childhood-onset (age $<17$ years) type 1 diabetes between 1950 and 1980 (and seen at Children's Hospital of Pittsburgh within 1 year of diagnosis), were first evaluated for EDC between 1986 and 1988, when mean age and diabetes duration $( \pm \mathrm{SD})$ were $28 \pm 7.9$ and $19 \pm 7.5$ years, respectively. Participants were followed biennially by survey, and for the first 10 years and again at 18 years by examination. Study protocols were approved by the University of Pittsburgh Institutional Review Board, and all participants provided written informed consent.

Clinical evaluation and procedures EDC participants completed surveys regarding medical history and demographic information prior to their clinical examination. Blood pressure was measured according to the Hypertension Detection and Follow-Up Program protocol with a random-zero sphygmomanometer after a 5 min rest. An ever smoker was defined as a person who had smoked $\geq 100$ cigarettes over their lifetime.

Fasting blood samples were taken to measure glycosylated haemoglobin $\left(\mathrm{HbA}_{1}\right)$, lipids and lipoproteins. For analysis purposes, original $\mathrm{HbA}_{1}$ values were converted to Diabetes Complications and Control Trial (DCCT)-aligned
$\mathrm{HbA}_{1 \mathrm{c}}$ using the following regression equation derived from duplicate assays: DCCT $\mathrm{HbA}_{1 \mathrm{c}}=0.14+0.83$ (EDC $\mathrm{HbA}_{1}$ ). Total cholesterol and triacylglycerol were measured enzymatically, and HDL-cholesterol (HDL-c) was determined using a heparin and manganese chloride precipitation method [12]. White blood cell (WBC) counts were obtained with a Coulter Counter (model S-plus IV, Beckman-Coulter, Fullerton, CA, USA), and fibrinogen using a biuret colorimetric procedure and a clotting method.

Height and weight were measured for body mass index (BMI, in kilogram per square meter), and waist and hip circumferences were measured at least twice and averaged to calculate WHR. Insulin sensitivity was estimated using a regression equation for glucose disposal rate (eGDR) derived from hyperinsulinaemic-euglycaemic clamp studies, as previously described [13].

Serum and urinary albumin were measured by immunonephelometry [14], and creatinine was assayed using an Ectachem 400 Analyzer (Eastman Kodak, Rochester, NY, USA). Estimated glomerular filtration rate (eGFR) was calculated using the Modification of Diet in Renal Disease (MDRD) equation [15]. Baseline renal damage status was defined on the basis of urinary AER in at least two of three timed urine collections as $<20 \mu \mathrm{g} / \mathrm{min}$-normoalbuminuria (NA), $20-200 \mu \mathrm{g} / \mathrm{min}$ - microalbuminuria (MA), or $>200 \mu \mathrm{g} / \mathrm{min}$ —overt nephropathy (ON). End stage renal disease (ESRD) was defined as renal failure or transplantation. Progression to renal disease during follow-up was defined as confirmed MA in two consecutive EDC cycles, or any ON or ESRD. Overall, 53 (8.1\%) participants, 38 of whom had NA at baseline, provided no updated renal examination data after the baseline examination.

Macrovascular disease was defined as myocardial infarction, stroke, coronary or peripheral revascularisation, angiographic stenosis $\geq 50 \%$, carotid surgery, or amputation of an ischaemic limb, confirmed by hospital records. Proliferative retinopathy was assessed using stereoscopic fundus photographs of fields 1, 2 and 4 with a Zeiss camera, read by the University of Wisconsin-Madison Fundus Photography Reading Center and classified based on the modified Arlie House system [16]. For participants refusing fundus photographs ( $n=$ 43), proliferative retinopathy was defined as receiving laser phototherapy for proliferative retinopathy.

Mortality data Vital status was determined as of 1 January 2008. Deaths were also identified via searches in both the Social Security Death Index and the National Death Index. Vital status could not be ascertained for $16(2.4 \%)$ participants. To confirm each death, death certificates were obtained, plus as many as possible of the following: (1) pertinent medical records; (2) autopsy/coroner's reports; and (3) interview with next of kin regarding the circumstances surrounding the death. The underlying cause of 
death, and rank order for all contributing causes, for each decedent were determined by a Mortality Classification Committee consisting of at least two physician epidemiologists using all available data based on standardised procedures [17].

Statistical analysis Each variable's distributional characteristics were assessed for normality. Student's $t$ test and oneway ANOVA were used to compare normally distributed variables between groups, while Mann-Whitney $U$ and Kruskal-Wallis tests were used for non-normally distributed variables, adjusting for multiple comparisons using the Bonferroni correction. The $\chi^{2}$ (or Fisher's exact) test was used to compare categorical variables between groups, as appropriate. Significant univariate measures and other variables known to be associated with mortality were made available in multivariable Cox proportional hazards regression models using backward selection. As age and diabetes duration are highly correlated in this cohort $(r=0.85)$, only duration was made available to multivariable models. The proportional hazards assumption was assessed visually and verified by testing time-dependent interaction variables. Survival plots by baseline albuminuria status, macrovascular disease status, and proliferative retinopathy status were produced based on these multivariable models. The effect of progression to RD on mortality was assessed as a timevarying covariate in a diabetes duration-adjusted Cox model for study participants with normoalbuminuria at baseline. With this method, participants were treated as normoalbuminuric until the examination when progression to $\mathrm{RD}$ occurred, and then considered as having RD thereafter.

Expected mortality was estimated using the person-years method based on general population life tables for Allegheny County, Pennsylvania [18]. Age, sex, and race-adjusted standardised mortality ratios were calculated as the observed divided by the expected number of deaths in each age, sex, and race category. Mortality rates were compared using rate ratio (RR) analyses and calculating 95\% CIs [19].

Hazard ratios (HR) for continuous variables are reported per increase of one SD. Statistical significance was considered as $p<0.05$. Analyses were completed using either SPSS 17.0 (SPSS, Chicago, IL, USA) or SAS 9.1 (SAS Institute, Cary, NC, USA). For comparability purposes, analyses, complication groupings, and covariates reflect those in Groop et al. [8], along with further covariates made available for modelling based on univariate analyses in this report, as described.

\section{Results}

Baseline characteristics by degree of renal disease and function At baseline, approximately half the population had
NA, while half had varying levels of RD (MA 21.3\%, ON $22.2 \%$, and ESRD $3.8 \%$; Table 1). Compared with individuals with NA, those with RD were significantly older and had longer duration of diabetes, greater WHR, and adverse cardiovascular (systolic blood pressure [SBP], diastolic blood pressure, non-HDL-c, HDL-c, triacylglycerol) and inflammatory (WBC, fibrinogen) risk profiles, which worsened in a linear fashion with severity of RD. No differences in RD status were seen by sex or race. Those with RD also had a higher prevalence of diabetes-related complications, such as proliferative retinopathy and macrovascular disease, which increased with greater renal damage. Similar trends were seen when the cohort was stratified by renal function measures (data not shown).

Mortality trends The median follow-up time was 20.1 years (range 0.2-21.7 years). Of 658 participants, 152 (23.1\%) died, giving an overall mortality of $1.3 / 100$ person-years (Table 2). Although mortality did not significantly differ by sex or race, mortality significantly increased with greater albumin excretion rate.

Association between renal disease and 10 year or 20 year mortality To approximately replicate the recently published FinnDiane results, we calculated 10 year specific HRs and SMRs according to baseline RD status (Table 3). The risk of death in individuals with NA at baseline was not significantly greater than that in the local age and sexmatched general population (SMR 1.3, 95\% CI 0.2-2.5); however, mortality increased stepwise with increasing renal damage, as did the SMR relative to the local general population.

Twenty-year survival curves generated using multivariable Cox regression analysis are illustrated by baseline stages of $\mathrm{RD}$, macrovascular disease, or proliferative retinopathy (Fig. 1). As can be seen, renal disease categories showed the greatest impact on survival. Survival curves did not differ when stratified by sex or after excluding individuals with ESRD (data not shown). Compared with those with NA at baseline, individuals with MA, ON, or ESRD had 20 year mortality risks that were 2.4 (95\% CI 1.4-4.3), 4.0 (2.3-7.0) or $9.0(4.3-18.7)$ times higher, respectively (Table 3 ). Other significant predictors of 20 year mortality that remained in the multivariable model included male sex (HR 1.7, 95\% CI 1.1-2.6), longer diabetes duration $(1.4,1.1-1.7)$, greater WHR $(1.5,1.2$ 1.9), higher $\mathrm{HbA}_{1 \mathrm{c}}$ level (1.3, 1.1-1.6), higher SBP (1.2, 1.0-1.4), positive smoking history $(1.9,1.3-2.7)$, MDRD eGFR $<60 \mathrm{ml} \mathrm{min}^{-1} 1.73 \mathrm{~m}^{-2}(1.8,1.1-2.9)$, and presence of macrovascular disease $(1.9,1.3-2.9)$. Other significant predictors of mortality at the 10 year follow-up did not dramatically differ from those at 20 years, except that baseline non-HDL-c replaced $\mathrm{HbA}_{1 \mathrm{c}}$ level in the model 
Table 1 Characteristics of the Pittsburgh EDC population by baseline renal damage categories

\begin{tabular}{|c|c|c|c|c|}
\hline Variable & $\begin{array}{l}\text { NA } \\
(\text { AER }<20 \mu \mathrm{g} / \mathrm{min})\end{array}$ & $\begin{array}{l}\text { MA } \\
\text { (AER } 20-200 \mu \mathrm{g} / \mathrm{min} \text { ) }\end{array}$ & $\begin{array}{l}\text { ON } \\
(\text { AER }>200 \mu \mathrm{g} / \mathrm{min})\end{array}$ & ESRD \\
\hline$n(\%)$ & $347(52.7)$ & $140(21.3)$ & $146(22.2)$ & $25(3.8)$ \\
\hline Male sex & $162(46.7)$ & $76(54.3)$ & $79(54.1)$ & $16(64.0)$ \\
\hline Black race & $7(2.0)$ & $3(2.1)$ & $3(2.1)$ & $2(8.0)$ \\
\hline Age (years) ${ }^{\mathrm{d}}$ & $25.3 \pm 7.7$ & $28.6 \pm 7.9^{\mathrm{b}}$ & $30.9 \pm 6.2^{b}$ & $36.1 \pm 5.8^{\mathrm{b}}$ \\
\hline Diabetes duration (years) ${ }^{\mathrm{d}}$ & $17.0 \pm 7.1$ & $20.5 \pm 7.3^{b}$ & $22.6 \pm 6.6^{\mathrm{b}}$ & $26.8 \pm 5.3^{b}$ \\
\hline BMI $\left(\mathrm{kg} / \mathrm{m}^{2}\right)$ & $23.2 \pm 3.2$ & $23.9 \pm 3.6$ & $23.9 \pm 3.0$ & $23.7 \pm 3.1$ \\
\hline WHR $^{\mathrm{d}}$ & $0.81 \pm 0.06$ & $0.84 \pm 0.07^{\mathrm{b}}$ & $0.84 \pm 0.08^{\mathrm{b}}$ & $0.90 \pm 0.07^{\mathrm{b}}$ \\
\hline Insulin dose $\left(\mathrm{U} \mathrm{kg}^{-1} \mathrm{day}^{-1}\right)$ & $0.80 \pm 0.23$ & $0.79 \pm 0.25$ & $0.75 \pm 0.25$ & $0.82 \pm 0.41$ \\
\hline $\mathrm{HbA}_{1 \mathrm{c}}(\%)$ & $8.56 \pm 1.39$ & $9.15 \pm 1.71^{\mathrm{b}}$ & $8.92 \pm 1.60$ & $8.96 \pm 1.44$ \\
\hline eGDR $\left(\mathrm{mg} \mathrm{kg}^{-1} \min ^{-1}\right)^{\mathrm{d}}$ & $8.56 \pm 1.33$ & $7.45 \pm 1.79^{\mathrm{b}}$ & $6.63 \pm 2.17^{\mathrm{b}}$ & $4.70 \pm 1.72^{\mathrm{b}}$ \\
\hline Serum creatinine $(\mu \mathrm{mol} / 1)^{\mathrm{d}}$ & $70.7(53.0-88.4)$ & $79.6(61.9-97.2)^{\mathrm{a}}$ & $88.4(70.7-123.8)^{\mathrm{b}}$ & $194.5(123.8-455.3)^{\mathrm{b}}$ \\
\hline MDRD eGFR $\left(\mathrm{ml} \mathrm{min} \operatorname{mos}^{-1} 1.73 \mathrm{~m}^{-2}\right)^{\mathrm{d}}$ & $111.9 \pm 44.3$ & $101.2 \pm 36.8^{\mathrm{a}}$ & $75.3 \pm 40.2^{\mathrm{b}}$ & $32.8 \pm 22.6^{\mathrm{b}}$ \\
\hline Systolic BP $(\mathrm{mmHg})^{\mathrm{d}}$ & $108.1 \pm 10.6$ & $112.4 \pm 12.9^{\mathrm{b}}$ & $124.0 \pm 16.7^{\mathrm{b}}$ & $139.8 \pm 30.3^{\mathrm{b}}$ \\
\hline Diastolic BP $(\mathrm{mmHg})^{\mathrm{d}}$ & $69.0 \pm 8.1$ & $73.1 \pm 10.5^{\mathrm{b}}$ & $80.2 \pm 11.9^{\mathrm{b}}$ & $84.3 \pm 15.9^{\mathrm{b}}$ \\
\hline BP medication use & $5(1.5)$ & $10(7.6)^{\mathrm{a}}$ & $42(30.2)^{\mathrm{b}}$ & $10(40.0)^{\mathrm{b}}$ \\
\hline Pulse (beats/min) & $76.6 \pm 9.3$ & $80.1 \pm 9.9^{b}$ & $81.4 \pm 10.0^{\mathrm{b}}$ & $75.4 \pm 13.0$ \\
\hline Total cholesterol $(\mathrm{mmol} / \mathrm{l})^{\mathrm{d}}$ & $4.58 \pm 0.84$ & $5.05 \pm 0.99^{\mathrm{b}}$ & $5.62 \pm 1.30^{\mathrm{b}}$ & $5.84 \pm 1.26^{\mathrm{b}}$ \\
\hline Non-HDL-c $(\mathrm{mmol} / \mathrm{l})^{\mathrm{d}}$ & $3.15 \pm 0.83$ & $3.66 \pm 0.96^{\mathrm{b}}$ & $4.27 \pm 1.33^{\mathrm{b}}$ & $4.61 \pm 1.24^{\mathrm{b}}$ \\
\hline HDL-c $(\mathrm{mmol} / \mathrm{l})^{\mathrm{c}}$ & $1.43 \pm 0.31$ & $1.37 \pm 0.31$ & $1.35 \pm 0.34^{\mathrm{a}}$ & $1.23 \pm 0.38^{\mathrm{a}}$ \\
\hline Triacylglycerol $(\mathrm{mmol} / \mathrm{l})^{\mathrm{d}}$ & $0.81(0.62-1.10)$ & $0.99(0.77-1.50)^{\mathrm{b}}$ & $1.18(0.85-1.87)^{\mathrm{b}}$ & $1.93(1.44-3.10)^{\mathrm{b}}$ \\
\hline WBC count $\left(\times 10^{9} / 1\right)^{\mathrm{d}}$ & $5.8(5.0-7.0)$ & $6.5(5.5-8.0)^{\mathrm{b}}$ & $6.9(5.8-8.4)^{\mathrm{b}}$ & $7.6(6.0-9.2)^{\mathrm{b}}$ \\
\hline Fibrinogen $(\mu \mathrm{mol} / 1)^{\mathrm{d}}$ & $7.4(6.2-8.8)$ & $8.2(7.1-9.8)^{\mathrm{b}}$ & $9.4(7.8-11.6)^{\mathrm{b}}$ & $9.1(7.9-11.0)^{\mathrm{b}}$ \\
\hline Proliferative retinopathy & $46(13.5)$ & $44(32.4)^{b}$ & $91(62.8)^{b}$ & $23(92.0)^{b}$ \\
\hline Macrovascular disease & $20(5.8)$ & $16(11.4)$ & $21(14.4)^{\mathrm{a}}$ & $11(44.0)^{\mathrm{b}}$ \\
\hline Ever smoker & $105(31.3)$ & $58(43.3)^{\mathrm{a}}$ & $71(48.6)^{b}$ & $14(56.0)^{\mathrm{a}}$ \\
\hline
\end{tabular}

Data presented as $n(\%)$ or mean $\pm \mathrm{SD}$ or median (interquartile range)

Comparison with participants with normoalbuminuria: ${ }^{\mathrm{a}} p<0.05,{ }^{\mathrm{b}} p<0.001$

${ }^{\mathrm{c}}$ Significant linear trend, $p<0.05,{ }^{\mathrm{d}}$ significant linear trend, $p<0.001$

(data not shown). Compared with the age and sex-matched general population, individuals with NA had twice as many deaths as would have been expected in the general population over 20 years of follow-up (SMR 2.0, 95\% CI 1.2-2.8). The SMRs increase steadily for individuals with increasingly severe RD: MA, 6.4 (4.4-8.4); ON, 12.5 (9.515.4); ESRD 29.8 (16.8-42.9).

Mortality in participants with normoalbuminuria We conducted a subgroup analysis of individuals with NA at baseline to determine how many progressed to RD during the study period, and whether progression to RD could explain their twofold increased mortality over the longer (20 year) follow-up period. Of the 347 individuals with NA at baseline, $64(18.4 \%)$ progressed to RD (MA, $n=33$; ON, $n=22$; ESRD, $n=9$ ) over a median follow-up of 17.9 years. For those who remained free of $\mathrm{RD}$, the median follow-up time was 18.2 years.
In the baseline NA group, 13 (4.6\%) individuals of those who remained free of $\mathrm{RD}(n=283)$ died during the 20 year period compared with $11(17.2 \%)$ of those who progressed to RD (type 1 diabetes duration-adjusted HR from timevarying Cox model $=4.3$, 95\% CI 1.6-12.1). After exclusion of the 64 individuals with NA at baseline who progressed to $\mathrm{RD}$, the adjusted 20 year age and sexmatched SMR for the NA group fell from 2.0 to 1.2 (95\% CI 0.5-1.9).

Comparison of cause of death by renal disease status We also compared the primary cause of death in those who consistently had NA with those with baseline NA who later developed RD during follow-up (Table 4). Those with RD had a longer duration of diabetes $(p<0.05)$ and, in $93 \%$ of cases, their death was primarily attributed to a diabetesrelated cause. In contrast, only $54 \%$ of deaths in the NA group were diabetes-related $(p=0.001)$. Of these seven 
Table 2 Mortality rates in type 1 diabetes participants by sex, race, and baseline renal damage categories

\begin{tabular}{|c|c|c|c|c|c|}
\hline Variable & $n(\%)$ & Median FUT (years) & Total FUT (years) & Deaths, $n(\%)$ & $\begin{array}{l}\text { Mortality rate }(95 \% \mathrm{CI} \text {; } \\
\text { per } 100 \text { person-years })\end{array}$ \\
\hline Overall & 658 & 20.1 & 11,870 & $152(23.1)$ & $1.3(1.1,1.5)$ \\
\hline \multicolumn{6}{|l|}{ Sex } \\
\hline Male & $333(50.6)$ & 20.0 & 5,922 & $85(26.2)$ & $1.4(1.1,1.7)$ \\
\hline Female & $325(49.4)$ & 20.2 & 5,948 & $67(21.1)$ & $1.1(0.9,1.4)$ \\
\hline \multicolumn{6}{|l|}{ Race } \\
\hline White & $643(97.7)$ & 20.1 & 11,610 & 147 (22.9) & $1.3(1.1,1.5)$ \\
\hline Black & $15(2.3)$ & 20.2 & 260 & $5(33.3)$ & $1.9(0.2,3.6)$ \\
\hline \multicolumn{6}{|l|}{ AER } \\
\hline Normoalbuminuria & $347(52.7)$ & 20.4 & 6,851 & $24(7.1)$ & $0.3(0.2,0.5)$ \\
\hline Microalbuminuria & $140(21.3)$ & 20.1 & 2,510 & $39(28.1)$ & $1.6(1.1,2.0)^{\mathrm{a}}$ \\
\hline Overt nephropathy & $146(22.2)$ & 19.0 & 2,275 & $69(47.3)$ & $3.0(2.3,3.8)^{\mathrm{a}, \mathrm{b}}$ \\
\hline ESRD & $25(3.8)$ & 8.6 & 234 & $20(80.0)$ & $8.6(4.8,12.3)^{\mathrm{a}, \mathrm{b}, \mathrm{c}}$ \\
\hline
\end{tabular}

$p<0.001$ for rate ratio compared with: ${ }^{\mathrm{a}}$ normoalbuminuria; ${ }^{\mathrm{b}}$ microalbuminuria; ${ }^{\mathrm{c}}$ overt nephropathy

FUT, follow-up time

diabetes-related deaths in the NA group, two were due to hypoglycaemia (one complicated by alcohol abuse), four due to cardiac disease, and one due to pneumonia (complicated by tobacco abuse and asthma).

\section{Discussion}

Our results confirm and expand, with longer follow-up, previously published findings by the FinnDiane group [8] demonstrating that, in the absence of renal disease, individuals with type 1 diabetes experience mortality rates similar to those in the general population. Thus, although the risk of death among participants with normoalbuminuria at study entry was twice the risk of death in the age and sex-matched general population after 20 years of follow-up, restricting analyses to individuals maintaining normoalbuminuria throughout the duration of the study produced mortality rates not dissimilar to those in the general population. Correspondingly, a much greater proportion of deaths was due to non-diabetes-related causes among those

Table 3 Ten and 20 year mortality risk and standardised mortality ratios in EDC participants with type 1 diabetes categorised by renal damage status at baseline, before and after adjustment for other risk factors

\begin{tabular}{|c|c|c|c|c|c|c|}
\hline Variable & Unadjusted HR & $95 \% \mathrm{CI}$ & Adjusted $\mathrm{HR}^{\mathrm{a}}$ & $95 \% \mathrm{CI}$ & Adjusted $\mathrm{SMR}^{\mathrm{b}}$ & $95 \% \mathrm{CI}$ \\
\hline \multicolumn{7}{|l|}{10 year follow-up } \\
\hline \multicolumn{7}{|l|}{ AER category } \\
\hline Normoalbuminuria (ref) & 1.0 & - & 1.0 & - & 1.3 & $(0.2,2.5)$ \\
\hline Microalbuminuria & 6.8 & $(2.4,19.1)$ & 3.5 & $(1.1,11.4)$ & 6.6 & $(3.0,10.1)$ \\
\hline Overt nephropathy & 16.3 & $(6.4,42.0)$ & 5.2 & $(1.6,16.1)$ & 14.2 & $(9.2,19.2)$ \\
\hline ESRD & 68.6 & $(25.1,187.6)$ & 28.8 & $(8.7,95.9)$ & 38.9 & $(19.8,57.9)$ \\
\hline \multicolumn{7}{|l|}{20 year follow-up } \\
\hline \multicolumn{7}{|l|}{ AER category } \\
\hline Normoalbuminuria (ref) & 1.0 & - & 1.0 & - & 2.0 & $(1.2,2.8)$ \\
\hline Microalbuminuria & 4.5 & $(2.7,7.5)$ & 2.4 & $(1.4,4.3)$ & 6.4 & $(4.4,8.4)$ \\
\hline Overt nephropathy & 9.3 & $(5.8,14.6)$ & 4.0 & $(2.3,7.0)$ & 12.5 & $(9.5,15.4)$ \\
\hline ESRD & 28.9 & $(15.9,52.6)$ & 9.0 & $(4.3,18.7)$ & 29.8 & $(16.8,42.9)$ \\
\hline
\end{tabular}

${ }^{\mathrm{a}}$ Variables available to the model: duration of diabetes, sex, race, waist to hip ratio, $\mathrm{HbA}_{1 \mathrm{c}}$, systolic $\mathrm{BP}$, diastolic $\mathrm{BP}$, $\mathrm{BP}$ medication use, high density lipoprotein cholesterol, non-high density lipoprotein cholesterol, WBC, estimated glomerular filtration rate, presence of macrovascular disease, presence of proliferative retinopathy, and ever smoker

${ }^{\mathrm{b}}$ Adjusted for age, race, and sex

ref, reference 

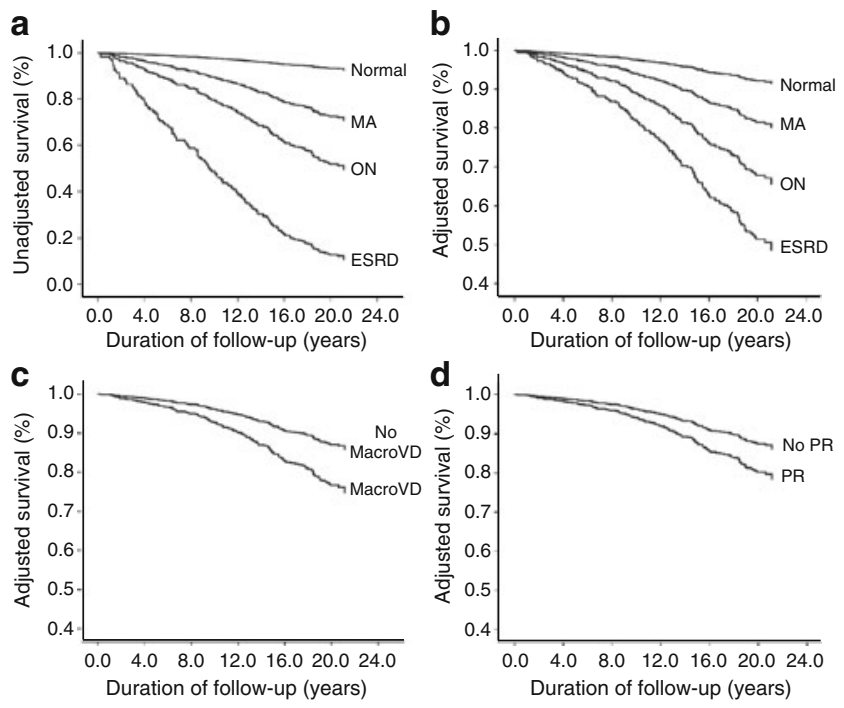

Fig. 1 Survival plots based on Cox-adjusted analysis of EDC participants stratified for the presence and severity of albuminuria, both unadjusted (a) and adjusted (b), the presence of macrovascular disease (c), and the presence of proliferative retinopathy (d) at baseline. Data shown in $\mathbf{b}-\mathbf{d}$ are adjusted for duration of diabetes, sex, race, waist to hip ratio, $\mathrm{HbA}_{1 \mathrm{c}}$, systolic $\mathrm{BP}$, renal function (MDRD eGFR), ever smoker, and the other complications not presented (i.e. macrovascular disease, proliferative retinopathy and/or renal damage). Other variables available to the model but not selected include: diastolic BP, BP medication use, high density lipoprotein cholesterol and non-high density lipoprotein cholesterol, and WBC count. Data shown in $\mathbf{d}$ are not adjusted for renal damage status (albuminuria category). PR, proliferative nephropathy; VD, vascular disease

who had remained normoalbuminuric, whereas individuals with renal disease died primarily from acute and chronic diabetes complications.

It has long been known that the development of overt nephropathy in type 1 diabetes carries excess mortality risk [17], although the importance of microalbuminuria has been less clear, both because of its often transient nature and also because its sensitivity in identifying those at risk of progressive renal function loss in diabetes has been questioned [20-22]. Nevertheless, we have previously shown that although moderately decreased estimated renal function (e.g. glomerular filtration rate) can occur in type 1 diabetes in the absence of preceding or concurrent albuminuria, this event is rare, particularly when the Cockcroft-Gault formula is used as opposed to the Modification of Diet in Renal Disease (MDRD) study formula to estimate renal function [23]. Moreover, the importance of microalbuminuria was further emphasised in a recent analysis of risk factors for major outcomes of type 1 diabetes, comprising the aggregate of coronary artery disease, renal failure and diabetes-related death, where it emerged as the single best predictor from all individual measures studied, including $\mathrm{HbA}_{1}$ [24]. In the present report, the presence of albuminuria at study entry was associated with an increased risk of mortality during follow-up. Indeed, mortality risk increased linearly with worsening of albuminuria, reaching, among those with ESRD, 30 times the mortality observed in the age and sexmatched general population.

Considerable focus has been placed on the search for potential mechanisms linking renal disease with excess mortality in diabetes, and a large body of literature has addressed the association between renal and cardiovascular disease. However, although renal damage (albuminuria) is a risk factor for cardiovascular outcomes, whether this relationship can be attributed to abnormalities caused by renal disease leading to atherosclerosis, or simply worsening of known cardiovascular risk factors, is unclear [24]. In addition, a further potential mechanism may reflect the 'common soil' hypothesis that both renal and cardiovascular states result from the same predisposing factor. In fact, insulin resistance has been proposed as one such factor underlying both renal and cardiovascular complications $[25,26]$, and thus could potentially explain much of the link between renal and cardiovascular outcomes. Nonetheless, we have previously shown that overt nephropathy is a strong independent risk factor for incident 'hard' coronary artery disease (CAD) events (but not total CAD), even after allowing for standard risk factors, including lipid and BP levels, as well as a measure of insulin resistance [25].

Of particular note is the finding that the vast majority of deaths in the renal disease group (93\%) were related to diabetes, whereas nearly half of the deaths in those without renal disease were clearly not associated with diabetes at all. It should be stressed that these cause of death

Table 4 Primary cause of death of Pittsburgh EDC study participants by most recent renal damage category

\begin{tabular}{lll}
\hline Variable & Normoalbuminuria & $\begin{array}{l}\text { Any renal } \\
\text { damage }\end{array}$ \\
\hline$n(\%)$ & $284(43.2)$ & $374(56.8)$ \\
$\begin{array}{l}\text { Overall deaths (\%) } \\
\text { Mean diabetes duration }\end{array}$ & $13(4.6)$ & $139(37.2)$ \\
$\quad$ at death & $30.3 \pm 8.9$ & $35.3 \pm 8.0^{*}$ \\
$\begin{array}{l}\text { Cause of death*** } \\
\text { Acute diabetes complications }\end{array}$ & $2(15.4)$ & \\
$\quad \begin{array}{l}\text { Chronic diabetes } \\
\quad \text { complications }\end{array}$ & $5(38.5)$ & $16(11.5)$ \\
$\quad \begin{array}{l}\text { Non-diabetes-related causes } \\
\text { c }\end{array}$ & $6(46.2)$ & $113(81.3)$ \\
$\quad$ Unknown & $0(0.0)$ & $9(6.5)$ \\
\hline
\end{tabular}

${ }^{a}$ Includes deaths from hypoglycaemia and diabetic ketoacidosis

${ }^{\mathrm{b}}$ Includes deaths from cardiovascular, renal, infectious and other diabetesrelated causes (e.g. diabetic gangrene, brain damage secondary to diabetes)

${ }^{\mathrm{c}}$ Includes deaths from accident, suicide and other non-diabetes-related causes (e.g. multiple sclerosis, gastrointestinal bleeding)

${ }^{*} p \leq 0.05, * * * p \leq 0.001$ 
classifications were made before these analyses were conceived and performed and thus no potential bias exists. However, half the deaths in the normoalbuminuric group appear to be diabetes related (due to acute and chronic diabetes complications). We thus cannot totally exclude a minor increased mortality risk in the absence of renal disease, although any such increase is likely to be very small. Interestingly, two of the seven 'diabetes-related' deaths in the NA group had significant concomitant conditions (alcohol abuse and asthma).

Although these results, along with the recently published findings from the FinnDiane study [8], are encouraging and suggest that prevention of kidney disease virtually eliminates the excess mortality associated with this diabetes type, analyses of complication rates over time within the EDC study demonstrate that much work still has to be done in preventing renal disease. For, while ESRD has declined dramatically and a declining trend in the incidence of overt nephropathy was also noted, this latter improvement was lost with longer follow-up, suggesting that advanced renal disease incidence is merely being delayed, not prevented [27]. In addition, CAD event rates have also not shown a fall after 30 years of follow-up [27], although a recent comparative analysis of the DCCT and EDC cohorts suggests that intensive therapy may reduce 30 year cardiovascular disease incidence [28]. Considerable effort, then, should be aimed at the control of modifiable risk factors, including hyperglycaemia, dyslipidaemia and hypertension.

The EDC study has several strengths for evaluating the relationship between renal disease and mortality, including its prospective design with biennial examinations to assess albuminuria status, its long-term follow-up to ascertain mortality, and its detailed classification of cause of death, including death certificates, autopsy and hospital records and review by an expert committee, using a standardised protocol to determine the primary cause of death and to rank contributory causes [17]. Ascertainment of numerous relevant demographic and clinical variables also allowed us to incorporate these into our analysis to minimise confounding. The longitudinal nature of the data also allows the assessment of the effect on mortality of progression from normoalbuminuria to renal disease. Renal disease classifications were based on multiple samples (and confirmed by persistence or progression at the next examination) using identical protocols throughout the study period. Our data are remarkably consistent with those previously published from the FinnDiane study, thus strengthening their overall validity, particularly given the different socioeconomic and healthcare backgrounds from which both cohorts derive [8, 29, 30].

However, this study is not without limitations. Our cohort is hospital-based; however, a comparison of the EDC population with the Allegheny County type 1 diabetes registry found the EDC cohort to be epidemiologically representative of the local type 1 diabetes population [11]. Also, the EDC population consists of individuals with longstanding diabetes. Thus, these data partially reflect outdated diabetes management and care, and may not be generalisable to individuals recently diagnosed with type 1 diabetes, whose experience may more closely resemble that of the DCCT intensive therapy cohort, as recently described [28].

In conclusion, our results demonstrate not only that, in long-term follow-up, renal disease, including persistent microalbuminuria, is a key determinant of long-term excess mortality in type 1 diabetes, but also that individuals with type 1 diabetes who avoid renal disease appear to achieve long-term mortality similar to, or only marginally higher than, that seen in the general population. These results and those of the FinnDiane study should help focus attention on the identification of those at risk of microalbuminuria [31] and the development of appropriate prevention strategies.

Acknowledgements The Pittsburgh EDC Study was supported by a grant from the National Institute of Diabetes and Digestive and Kidney Diseases (R01-DK034818). AMS was supported by a training grant from the National Institute of Diabetes and Digestive and Kidney Diseases (F30-DK082137). The authors also acknowledge the long-term help of the EDC participants, and J. Sanders (University of Pittsburgh, Pittsburgh, PA, USA) for assistance with manuscript preparation.

Duality of interest The authors declare that there is no duality of interest associated with this manuscript.

\section{References}

1. Asao K, Sarti C, Forsen T et al (2003) Long-term mortality in nationwide cohorts of childhood-onset type 1 diabetes in Japan and Finland. Diabetes Care 26:2037-2042

2. Nishimura R, LaPorte RE, Dorman JS, Tajima N, Becker D, Orchard TJ (2001) Mortality trends in type 1 diabetes. The Allegheny County (Pennsylvania) Registry 1965-1999. Diabetes Care 24:823-827

3. Rossing P, Hougaard P, Borch-Johnsen K, Parving HH (1996) Predictors of mortality in insulin dependent diabetes: 10 year observational follow up study. BMJ 313:779-784

4. Messent JW, Elliott TG, Hill RD, Jarrett RJ, Keen H, Viberti GC (1992) Prognostic significance of microalbuminuria in insulindependent diabetes mellitus: a twenty-three year follow-up study. Kidney Int 41:836-839

5. Forsblom CM, Groop PH, Ekstrand A, Groop LC (1992) Predictive value of microalbuminuria in patients with insulindependent diabetes of long duration. BMJ 305:1051-1053

6. Nathan DM, Cleary PA, Backlund JY et al (2005) Intensive diabetes treatment and cardiovascular disease in patients with type 1 diabetes. N Engl J Med 353:2643-2653

7. Hovind P, Tarnow L, Rossing K et al (2003) Decreasing incidence of severe diabetic microangiopathy in type 1 diabetes. Diabetes Care 26:1258-1264

8. Groop PH, Thomas MC, Moran JL et al (2009) The presence and severity of chronic kidney disease predicts all-cause mortality in type 1 diabetes. Diabetes 58:1651-1658 
9. Orchard TJ, Dorman JS, Maser RE et al (1990) Factors associated with avoidance of severe complications after $25 \mathrm{yr}$ of IDDM. Pittsburgh Epidemiology of Diabetes Complications Study I. Diabetes Care 13:741-747

10. Orchard TJ, Dorman JS, Maser RE et al (1990) Prevalence of complications in IDDM by sex and duration. Pittsburgh Epidemiology of Diabetes Complications Study II. Diabetes 39: $1116-1124$

11. Wagener DK, Sacks JM, LaPorte RE, Macgregor JM (1982) The Pittsburgh study of insulin-dependent diabetes mellitus. Risk for diabetes among relatives of IDDM. Diabetes 31:136-144

12. Warnick GR, Albers JJ (1978) Heparin-Mn2+ quantitation of high-density-lipoprotein cholesterol: an ultrafiltration procedure for lipemic samples. Clin Chem 24:900-904

13. Williams KV, Erbey JR, Becker D, Arslanian S, Orchard TJ (2000) Can clinical factors estimate insulin resistance in type 1 diabetes? Diabetes 49:626-632

14. Ellis D, Coonrod BA, Dorman JS et al (1989) Choice of urine sample predictive of microalbuminuria in patients with insulindependent diabetes mellitus. Am J Kidney Dis 13:321-328

15. Levey AS, Coresh J, Greene $\mathrm{T}$ et al (2007) Expressing the Modification of Diet in Renal Disease Study equation for estimating glomerular filtration rate with standardized serum creatinine values. Clin Chem 53:766-772

16. Early Treatment of Diabetic Retinopathy Study Coordinating Center (1980) Manual of operations. University of Maryland School of Medicine, Baltimore

17. Diabetes Epidemiology Research International Mortality Study Group (1991) International evaluation of cause-specific mortality and IDDM. Diabetes Care 14:55-60

18. Epidemiologic Query and Mapping System (EpiQMS) (2010) Bureau of Health Statistics, Pennsylvania Department of Health. Available from http://app2.health.state.pa.us/epiqms, accessed 17 March 2010

19. Armstrong BG (1995) Comparing standardized mortality ratios. Ann Epidemiol 5:60-64

20. Retnakaran R, Cull CA, Thorne KI, Adler AI, Holman RR (2006) Risk factors for renal dysfunction in type 2 diabetes: U.K. Prospective Diabetes Study 74. Diabetes 55:1832-1839

21. Kramer HJ, Nguyen QD, Curhan G, Hsu CY (2003) Renal insufficiency in the absence of albuminuria and retinopathy among adults with type 2 diabetes mellitus. JAMA 289:3273-3277
22. Molitch ME, Steffes M, Sun W et al (2010) The development and progression of renal insufficiency with and without albuminuria in adults with type 1 diabetes mellitus in the diabetes control and complications trial and epidemiology of diabetes interventions and complications study. Diabetes Care 33:1536-1543

23. Costacou T, Ellis D, Fried L, Orchard TJ (2007) Sequence of progression of albuminuria and decreased GFR in persons with type 1 diabetes: a cohort study. Am J Kidney Dis 50:721-732

24. Pambianco G, Costacou T, Orchard TJ (2007) The prediction of major outcomes of type 1 diabetes: a 12-year prospective evaluation of three separate definitions of the metabolic syndrome and their components and estimated glucose disposal rate: the Pittsburgh Epidemiology of Diabetes Complications Study experience. Diabetes Care 30:1248-1254

25. Orchard TJ, Olson JC, Erbey JR et al (2003) Insulin resistancerelated factors, but not glycemia, predict coronary artery disease in type 1 diabetes: 10-year follow-up data from the Pittsburgh Epidemiology of Diabetes Complications Study. Diabetes Care 26:1374-1379

26. Orchard TJ, Chang YF, Ferrell RE, Petro N, Ellis DE (2002) Nephropathy in type 1 diabetes: a manifestation of insulin resistance and multiple genetic susceptibilities? Further evidence from the Pittsburgh Epidemiology of Diabetes Complication Study. Kidney Int 62:963-970

27. Pambianco G, Costacou T, Ellis D, Becker DJ, Klein R, Orchard TJ (2006) The 30-year natural history of type 1 diabetes complications: the Pittsburgh Epidemiology of Diabetes Complications Study experience. Diabetes 55:1463-1469

28. Nathan DM, Zinman B, Cleary PA et al (2009) Modern-day clinical course of type 1 diabetes mellitus after 30 years' duration: the diabetes control and complications trial/epidemiology of diabetes interventions and complications and Pittsburgh epidemiology of diabetes complications experience (1983-2005). Arch Intern Med 169:1307-1316

29. Arun CS, Stoddart J, Mackin P, MacLeod JM, New JP, Marshall SM (2003) Significance of microalbuminuria in long-duration type 1 diabetes. Diabetes Care 26:2144-2149

30. Allen KV, Walker JD (2003) Microalbuminuria and mortality in long-duration type 1 diabetes. Diabetes Care 26:2389-2391

31. Vergouwe Y, Soedamah-Muthu SS, Zgibor J et al (2010) Progression to microalbuminuria in type 1 diabetes: development and validation of a prediction rule. Diabetologia 53:254-262 